With the increasing use of antireflux surgery in the treatment of gastroesophageal reflux disease, the need has arisen for an accurate method of assessing the length of the esophagus. The enigmatic finding of short esophagus is a continuing matter of controversy with regard to prevalence, cause, and management. Short esophagus precludes a tension-free intra-abdominal antireflux procedure. The most effective treatment for the condition is an esophagus-lengthening procedure using a Collis gastroplasty. Alternative procedures, such as intrathoracic fundoplication or esophageal resection, are not widely accepted. The risk of encountering a short esophagus requiring a Collis gastroplasty is significantly greater in the presence of paraesophageal hernia, Barrett's esophagus, an esophageal stricture, and repeat surgery [1]. Esophagography has been reported to have a sensitivity of $66 \%$ and a positive predictive value of $27 \%$ for diagnosing short esophagus, whereas the length as measured by manometry had a sensitivity of $43 \%$ and a positive predictive value of $25 \%$ [2]. Intraoperative techniques have also been described that combine laparoscopic with endoscopic methods to determine the position of the gastroesophageal junction. The length of the intra-abdominal esophagus is determined by using a probe to measure the distance between the esophagogastric junction and the crura of the diaphragm [3]. Recurrent paraesophageal hernia is the most challenging scenario for repeat laparoscopic surgery, and the one most likely to require conversion to an open procedure. Some $30-80 \%$ of patients with paraesophageal hernias $5 \mathrm{~cm}$ or larger require gastroplasty $[4,5]$.

Between 1 January 2001 and 31 August 2003, we marked the Z-line with endoscopic clips (Olympus HX-200L-135) in eight patients in order to detect a potential short esophagus due to a large recurrent paraesophageal hernia. Immediately after clipping, an upright contrast study was carried out. The length of the esophagus, the distance between the Z-line and

\title{
Value of Endoscopic Marking of the Z-Line for Detecting Short Esophagus Before Repeat Surgery for Recurrent Paraesophageal Hernias
}

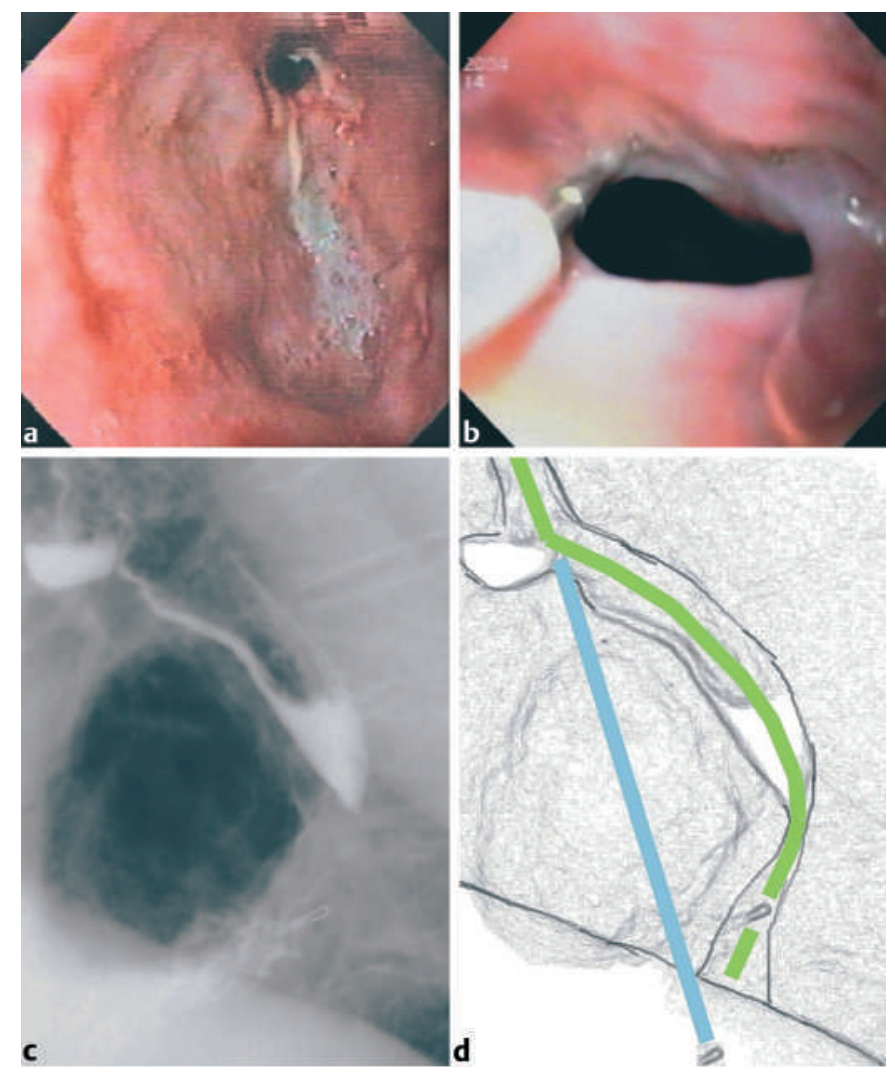

Figure 1 a A large recurrent paraesophageal hernia with an esophageal diverticulum. b Endoscopic clipping of the Z-line. c The precise location of the Z-line and the fact that the esophagus is appropriately long are well seen on this lateral esophagram. d The expected position of the Z-line after surgery.

the diaphragm, and the expected position of the esophagogastric junction after the operation were assessed on sagittal and lateral esophagrams (Figure [1]). With this method, it was predicted for three patients that it would not be possible to pull the esophagogastric junction at least $2 \mathrm{~cm}$ below the crura of the diaphragm. In these cases, a Collis gastroplasty was carried out (Figure [2]). In the other five patients, the method made it possible to exclude the possibility of a short esophagus, and this conclusion was confirmed by the findings during redo surgery. No recurrences were observed during a 1 -year follow-up period.

We consider that this method is appropriate and can be recommended not only before surgery for recurrent paraesophageal hernia, but in all pathological conditions in which the possibility of a short esophagus may arise.

\author{
Á. Altorjay', B. Paál', A. Horváth², \\ L. Horváth ${ }^{2}$, M. Ükös ${ }^{3}$, Z. Than ${ }^{3}$ \\ ${ }^{1}$ Dept. of Surgery \\ 2 Dept. of Gastroenterology \\ ${ }^{3}$ Dept. of Radiology, St. George University \\ Teaching Hospital, Székesfehérvár, \\ Hungary.
}

References

${ }^{1}$ Urbach DR, Khajanchee YS, Glasgow REet al. Preoperative determinants of an esophageal lengthening procedure in laparoscopic antireflux surgery. Surg Endosc 2001; 15: 1408-1412

${ }^{2}$ Mittal SK, Awad ZT, Tasset Met al. The preoperative predictability of the short esophagus in patients with stricture or paraesophageal hernia. Surg Endosc 2000; 14: 464-468 
${ }^{3}$ Awad ZT, Dickason TJ, Filipi CJet al. A combined laparoscopic-endoscopic method of assessment to prevent the complications of short esophagus. Surg Endosc 1999; 13: 626-627

${ }^{4}$ Dutta S, Bamehriz F, Boghossian Tet al. Outcome of laparoscopic redo fundoplication. Surg Endosc 2004; 18: 440-443

${ }^{5}$ Maziak DE, Todd TR, Pearson FG. Massive hiatus hernia: evaluation and surgical management. J Thorac Cardiovasc Surg 1998; 115: 53-62

\section{Corresponding Author}

\section{Á. Altorjay, M.D., Ph.D.}

\section{Dept. of Surgery}

St. George University Teaching Hospital Seregélyesi u. 3

8000 Székesfehérvár Hungary

Fax: $\quad+36-22-504-100$

E-mail: altorjay@mail.fmkorhaz.hu
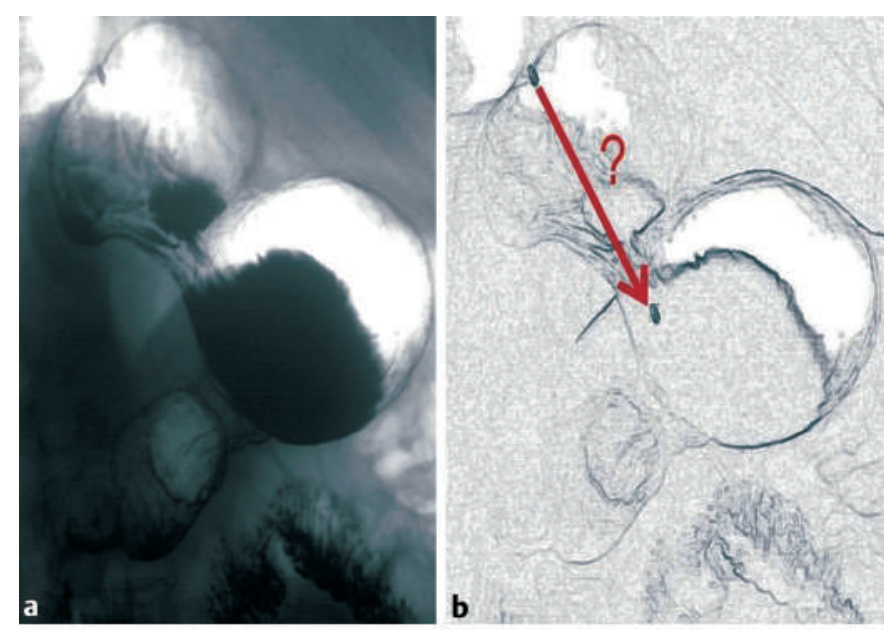

Figure 2 a,b A large paraesophageal hernia, with a definite short esophagus. c,d The esophagram after a Collis gastroplasty, with the lengthening of the esophagus by approximately $6 \mathrm{~cm}$ marked.

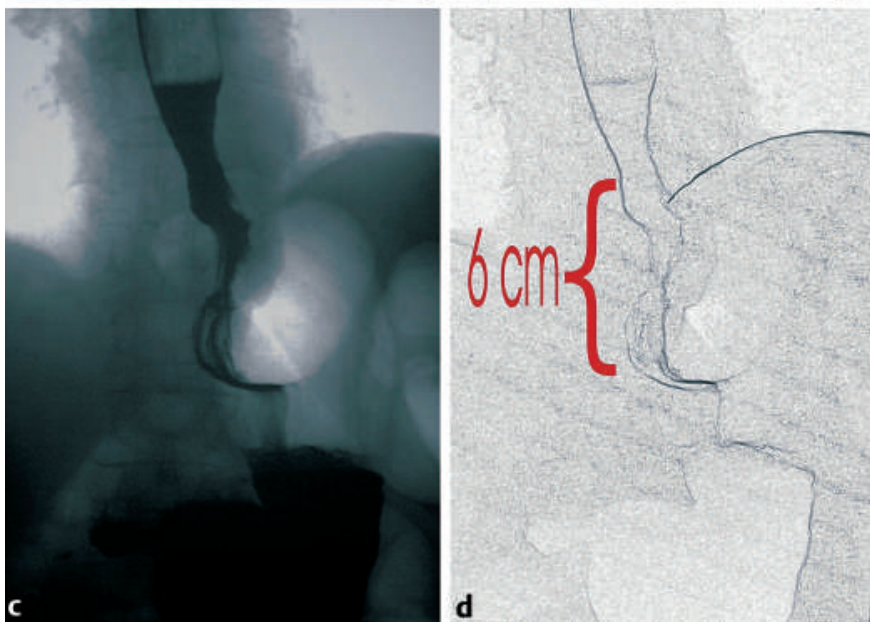

